\title{
AaWRKY17, a positive regulator of artemisinin biosynthesis, is involved in resistance to Pseudomonas syringae in Artemisia annua
}

\author{
Tiantian Chen', Yongpeng Li', Lihui Xie', Xiaolong Hao ${ }^{2}$, Hang Liu', Wei Qin', Chen Wang ${ }^{1}$, Xin Yan', \\ Kuanyu Wu-Zhang ${ }^{1}$, Xinghao Yao ${ }^{1}$, Bowen Peng ${ }^{1}$, Yaojie Zhang ${ }^{1}$, Xueqing Fu', Ling Li' and Kexuan Tang ${ }^{1 凶}$
}

\begin{abstract}
Artemisia annua, a traditional Chinese medicinal plant, remains the only plant source for artemisinin production, yet few genes have been identified to be involved in both the response to biotic stresses, such as pathogens, and artemisinin biosynthesis. Here, we isolated and identified the WRKY transcription factor (TF) AaWRKY17, which could significantly increase the artemisinin content and resistance to Pseudomonas syringae in A. annua. Yeast one-hybrid $(Y 1 H)$, dual-luciferase (dual-LUC), and electrophoretic mobility shift assay (EMSA) results showed that AaWRKY17 directly bound to the W-box motifs in the promoter region of the artemisinin biosynthetic pathway gene amorpha4,11-diene synthase (ADS) and promoted its expression. Real-time quantitative PCR (RT-qPCR) analysis revealed that the transcript levels of two defense marker genes, Pathogenesis-Related 5 (PR5) and NDR1/HIN1-LIKE 10 (NHL10), were greatly increased in AaWRKY17-overexpressing transgenic A. annua plants. Additionally, overexpression of AaWRKY17 in $A$. annua resulted in decreased susceptibility to $P$. syringae. These results indicated that AaWRKY17 acted as a positive regulator in response to $P$. syringae infection. Together, our findings demonstrated that the novel WRKY transcription factor AaWRKY17 could potentially be used in transgenic breeding to improve the content of artemisinin and pathogen tolerance in $A$. annua.
\end{abstract}

\section{Introduction}

Plants are exposed to various stresses from the environment during their lifecycle, including abiotic stresses such as drought and biotic stresses such as pathogens ${ }^{1,2}$. For survival and the continuation of the next generation, plants have adopted many defense mechanisms against biotic and abiotic stresses ${ }^{3}$. Among the various biotic stresses, pathogens are considered major threats to plant growth, development, and yield. To cope with pathogenic

\footnotetext{
Correspondence: Kexuan Tang (kxtang@sjtu.edu.cn)

${ }^{1} J o i n t$ International Research Laboratory of Metabolic and Developmental

Sciences, Frontiers Science Center for Transformative Molecules, Plant Biotechnology Research Center, Fudan-SJTU-Nottingham Plant Biotechnology R\&D Center, School of Agriculture and Biology, Shanghai Jiao Tong University, Shanghai 200240, China

2Laboratory of Medicinal Plant Biotechnology, College of Pharmacy, Zhejiang Chinese Medical University, Hangzhou 310053, China

These authors contributed equally: Tiantian Chen, Yongpeng L
}

stress, plants have developed sophisticated innate immunity pathways, including pathogen-associated molecular pattern-triggered immunity and effector-triggered immunity, which help them avoid greater pathogenic invasion $^{4-6}$.

The WRKY family forms a transcriptional network that regulates the complex signaling network in the plant defense system against pathogen infection ${ }^{7}$. As one of the largest TF families in plants, WRKY proteins are identified by two highly conserved domains: the amino acid motif WRKYGQK at the $\mathrm{N}$-terminus and a $\mathrm{C}_{2} \mathrm{H}_{2}$ or $\mathrm{C}_{2} \mathrm{HC}$ zinc-finger motif at the $\mathrm{C}$-terminus. Moreover, WRKY TFs can regulate the expression level of downstream target genes by directly binding to W-boxes (TTGAC(C/ $\mathrm{T})$ ) in their promoter regions ${ }^{8}$. Extensive research has shown that WRKY TFs play key roles in plant resistance to several pathogenic bacterial species? ${ }^{9}$. In plants,

\section{(c) The Author(s) 2021}

(c) (i) Open Access This article is licensed under a Creative Commons Attribution 4.0 International License, which permits use, sharing, adaptation, distribution and reproduction in any medium or format, as long as you give appropriate credit to the original author(s) and the source, provide a link to the Creative Commons license, and indicate if changes were made. The images or other third party material in this article are included in the article's Creative Commons license, unless indicated otherwise in a credit line to the material. If material is not included in the article's Creative Commons license and your intended use is not permitted by statutory regulation or exceeds the permitted use, you will need to obtain permission directly from the copyright holder. To view a copy of this license, visit http://creativecommons.org/licenses/by/4.0/. 
Pseudomonas syringae ${ }^{10}$, Botrytis cinerea ${ }^{11}$, Sclerotinia sclerotiorum $^{12}$, and Ralstonia solanacearum ${ }^{13}$ are the most common pathogens and have been well studied over the past decades. It is worth noting that many WRKY genes have been shown to enhance resistance to multiple pathogens. For example, Arabidopsis thaliana WRKY8, WRKY3, and WRKY4 enhanced resistance to $P$. syringae and B. cinerea infection ${ }^{14,15}$.

Artemisinin, a sesquiterpene lactone endoperoxide, is specifically synthesized in glandular trichomes ${ }^{16,17}$ and isolated from the traditional Chinese medicinal plant $A$. annua. It is well known that artemisinin is the best therapeutic agent against malaria and is also effective in the treatment of several cancers ${ }^{18-20}$. The artemisinin biosynthetic pathway has been extensively elucidated, and amorpha-4,11-diene synthase (ADS), Cyt P450-dependent hydroxylase (CYP71AV1), artemisinic aldehyde _11(13) reductase (DBR2), and aldehyde dehydrogenase 1 $(A L D H 1)$ are the key genes that encode enzymes catalyzing artemisinin production ${ }^{21}$. In addition to these structural genes, several lines of evidence have suggested that TFs play pivotal roles in artemisinin biosynthesis ${ }^{22-24}$. However, limited information is available about the role of A. аппиа TFs in biotic stress defense. Currently, A. апnиа is the only plant source for artemisinin production. However, the artemisinin content in wild-type (WT) $A$. annua was low. Therefore, it is important to develop $A$. annua germplasms with high yield and quality. Several studies have indicated that WRKY family members are involved in artemisinin production. For instance, AaWRKY1 is a positive regulator of $A D S$ and CYP71AV1 ${ }^{25}$. AaGSW1 (GLANDULAR TRICHOMESPECIFIC WRKY 1) was reported to increase artemisinin biosynthesis by directly binding to the promoter of CYP71AV ${ }^{26}$. However, few researchers have been able to identify any WRKY TFs that are involved in the regulation of plant resistance to some biotic stresses in A. annua.

In this study, we isolated a new WRKY TF, AaWRKY17, which positively regulates the transcription of $A D S$ by directly binding to its W-box motifs in the promoter, thus resulting in an increased artemisinin content. In addition, we demonstrated that the expression of AaWRKY17 was significantly induced by treatment with salicylic acid (SA), methyl jasmonate (MeJA), and P. syringae infection. Many phytohormones, such as $\mathrm{SA}, \mathrm{JA}^{27}, \mathrm{ABA}$, and $\mathrm{ET}^{28,29}$, can mediate the signaling pathways of plant responses to biotic stresses, and the expression of WRKY genes during plant defense responses also has a close relationship with these signaling pathways ${ }^{30}$. AtWRKY28 and AtWRKY75 mainly activate the JA/ET pathway to defend $A$. thaliana against $S$. sclerotiorum ${ }^{12}$. AtWRKY18, through activating $P R$ gene expression in the SA pathway ${ }^{31}$, enhances the resistance of $A$. thaliana to $P$. syringae. Moreover, we showed that overexpression of AaWRKY17 significantly increased the transcript levels of the defense marker genes $P R 5^{27,32}$ and $N H L 1 O^{33}$ and enhanced the resistance of $A$. annua to $P$. syringae. In conclusion, our research reveals a novel WRKY TF with dual functions in both artemisinin biosynthesis and biotic stress defense in $A$. annua, which can potentially be used in developing high-yielding and pathogen-resistant $A$. annua.

\section{Results}

\section{Cloning and characterization of AaWRKY17}

There are 135 WRKY genes in A. annua. Based on the transcriptome data, five WRKY TFs with high RPKM (reads per kilobase per million reads) values in trichomes were selected (Fig. 1): Aannua00069S018960, Aannua00977S163810, Aапnua04258S435660, Aannua06285S542770, and Aannua01956S266050. RT-qPCR results of different tissues showed that Aannua01956S266050 exhibited the highest expression level in trichomes among the five candidate genes, which was consistent with the transcriptome data from different $A$. annua tissues (Fig. 2A and Supplementary Fig. S1). Since artemisinin is only produced in trichomes of $A$. апnua, we speculated that Aannua01956S266050 potentially participated in artemisinin biosynthesis. We named the gene Aannua01956S266050 AaWRKY17 for further study according to the close relationship with AtWRKY17 in A. thaliana (Supplementary Fig. S2).

\section{Expression pattern and subcellular localization of AaWRKY17}

The expression pattern of AaWRKY17 in different tissues showed that it was highly expressed in trichomes (Fig. 2A). The expression profiling of AaWRKY17 in leaves at different positions showed that AaWRKY17 exhibited a stable expression level during the development of $A$. annua leaves (Fig. 2B). To further elucidate the expression pattern of $A a W R K Y 17$, we conducted AaWRKY17 promoter-driven $\beta$-glucuronidase (GUS)

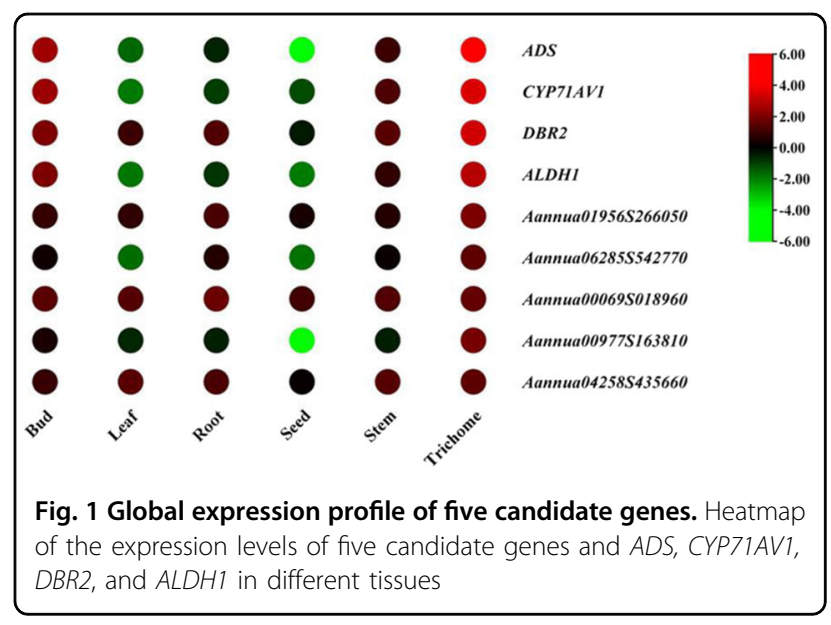


A

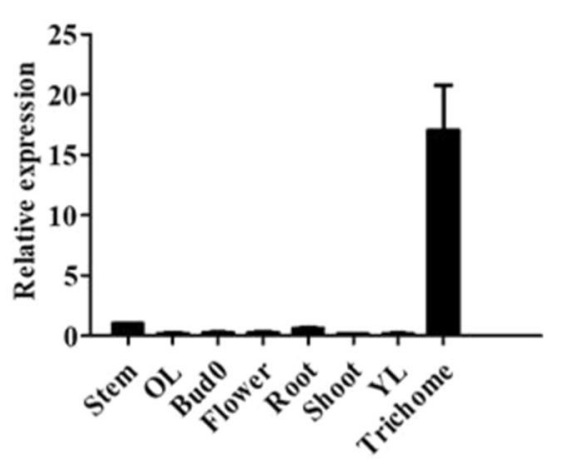

B

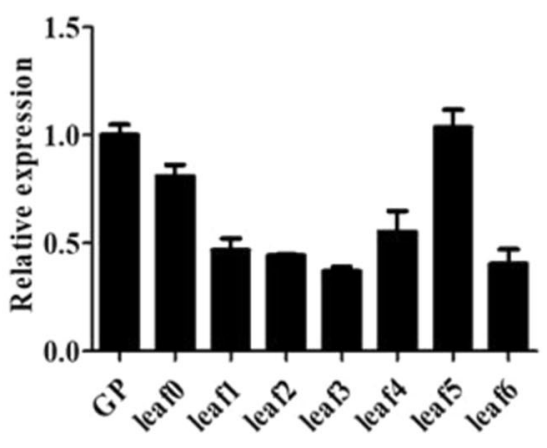

C
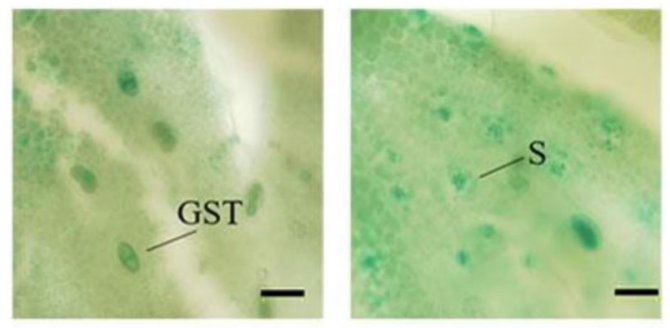

D

YFP

Bright
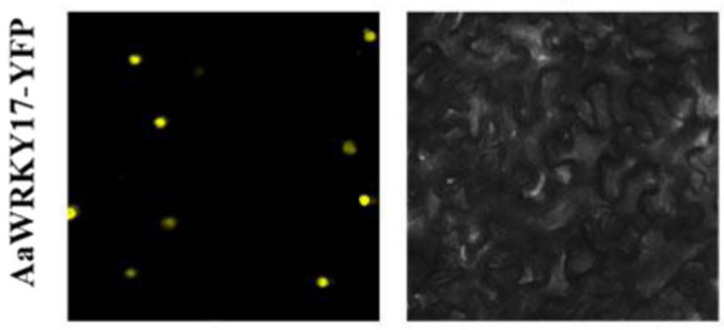

DAPI
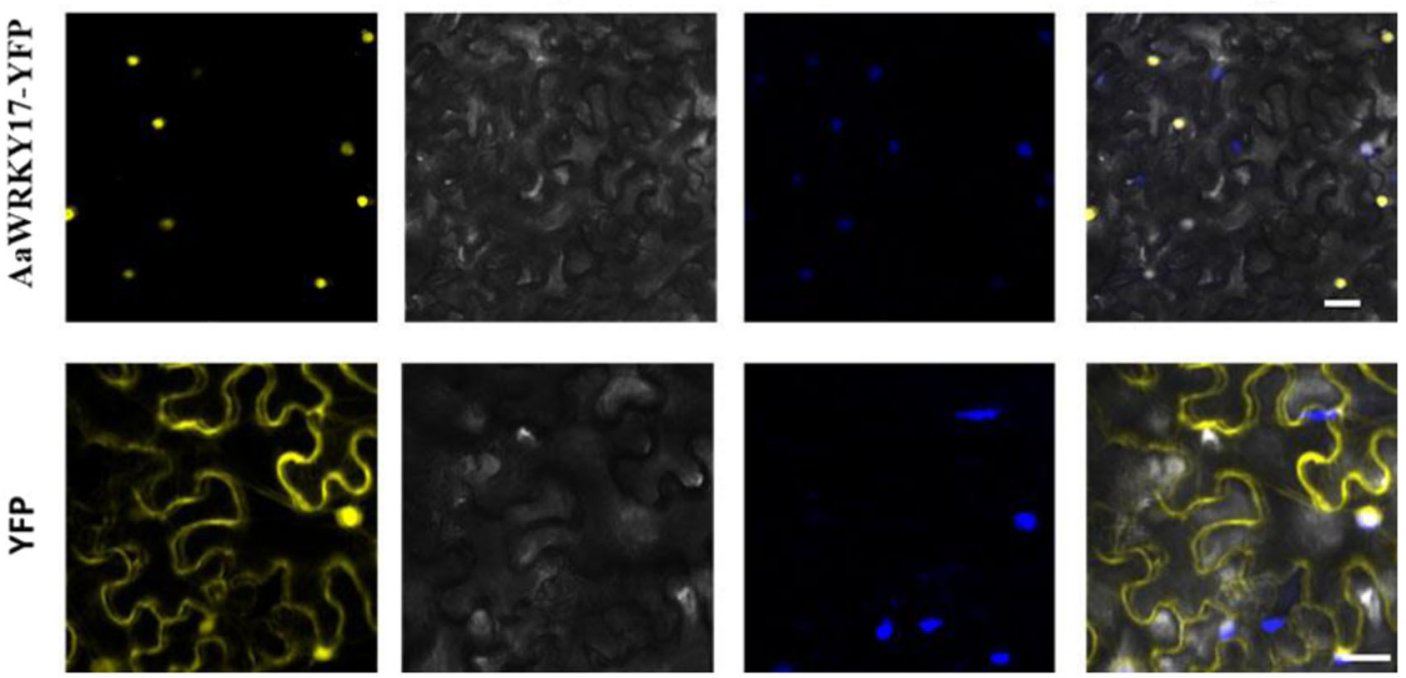

Fig. 2 Characterization of AaWRKY17. A The expression level of AaWRKY17 in different tissues of A. annua. B The expression level of AaWRKY17 at different positions of $A$. annua leaves. A, B were measured by RT-qPCR, and actin was used as an internal reference. Data are given as the mean \pm SD $(n=3)$. C AaWRKY 17 promoter-GUS (pAaWRKY17-GUS) expression in the young leaves of A. annua. GST glandular secreting trichome, $S$ stoma. Bars: $20 \mu \mathrm{m}$. D Subcellular localization of 35S:AaWRKY17-YFP in tobacco leaf epidermal cells. Yellow, yellow fluorescent protein (YFP). Blue, 4', 6-diamidino2-phenylindole staining (DAPI). Bars: $30 \mu \mathrm{m}$

transformation of A. annua. We observed that GUS was strongly detected in the glandular trichomes and stomas (Fig. 2C). To investigate the subcellular localization of AaWRKY17 in vivo. The AaWRKY17 protein was fused with the Yellow fluorescent protein (YFP) protein and transiently expressed in tobacco leaves. YFP fluorescence was detected in the nucleus (Fig. 2D), which indicated that AaWRKY17 might function as a transcription factor. 
Overexpression of AaWRKY17 in A. annua increases artemisinin biosynthesis, and downregulation of AaWRKY17 decreases artemisinin biosynthesis

To test whether AaWRKY17 is a regulator of artemisinin biosynthesis, we overexpressed AaWRKY17 in A. annua and selected the transgenic lines AaWRKY17-OE-1, AaWRKY17-OE-2, AaWRKY17-OE-3, and AaWRKY17OE-4 for further study according to their high expression levels of AaWRKY17. Compared with the WT plants, the transcript level of $A a W R K Y 17$ was increased 2.3- to 13.6fold in the transgenic lines AaWRKY17-OE-1, AaWRKY17-OE-2, AaWRKY17-OE-3, and AaWRKY17OE-4 (Fig. 3A). The leaves of 5-month-old AaWRKY17overexpressing transgenic plants were collected to measure artemisinin content using high-performance liquid chromatography (HPLC). In AaWRKY17-overexpressing lines, the artemisinin contents were significantly increased by $49.5-87.4 \%$ (Fig. 3E). To further prove the function of $A a W R K Y 17$ in the regulation of artemisinin biosynthesis in $A$. annua, we downregulated its expression by the RNA antisense approach. The transcript level of AaWRKY17 was suppressed to 54-68\% of the WT plant level in the selected AaWRKY17 antisense transgenic plants AaWRKY17-AS-1, AaWRKY17-AS-2, AaWRKY17AS-3, and AaWRKY17-AS-4 (Fig. 3B). Compared with the WT plants, the contents of artemisinin in AaWRKY17 antisense lines were decreased by $14.7-20.6 \%$ (Fig. 3F). In addition, we detected the transcript levels of four key enzyme genes of the artemisinin biosynthetic pathway, including $A D S, C Y P 71 A V 1, D B R 2$, and $A L D H 1$. The expression level of $A D S$ was increased 2.3- to 8.5fold in the AaWRKY17-overexpressing transgenic lines AaWRKY17-OE-1, AaWRKY17-OE-2, AaWRKY17-OE-3, and AaWRKY17-OE-4 (Fig. 3C). In the AaWRKY17 antisense transgenic lines AaWRKY17-AS-1, AaWRKY17-AS2, AaWRKY17-AS-3, and AaWRKY17-AS-4, the transcript level of $A D S$ was suppressed to $60-83 \%$ of the control level (Fig. 3D). However, no visible expression changes in CYP71AV1,DBR2, and $A L D H 1$ were observed in either AaWRKY17-overexpressing or AaWRKY17 antisense lines. These results indicated that AaWRKY17 might be a positive regulator of artemisinin biosynthesis by activating the expression of $A D S$.

\section{AaWRKY17 directly binds to and activates the promoter of ADS}

Since AaWRKY17-overexpressing lines showed increased expression of $A D S$, we next investigated the regulatory relationship between AaWRKY17 and ADS. Dual-luciferase (dual-LUC) assay results showed that AaWRKY17 could significantly activate the ADS promoter (Fig. 4A). Previous studies have reported that WRKY domains can specifically bind to W-box sequences in the promoter region of target genes ${ }^{8}$. Four W-boxes were found by analyzing the promoter sequence of $A D S$ (Supplementary Fig. S3). To test whether AaWRKY17 activates the expression of $A D S$ in a direct manner, $\mathrm{Y} 1 \mathrm{H}$ and EMSAs were performed. As shown in Fig. 4B, AaWRKY17 bound to two W-box motifs in the promoter region of $A D S$ (W2 and W4 boxes). Additionally, the direct binding activity of AaWRKY17 on the W2 box and W4 box in the $A D S$ promoter was further verified by EMSA experiments (Fig. 4C, D). These results revealed that AaWRKY17 was a positive regulator of artemisinin biosynthesis by directly activating the expression of $A D S$ in A. annua.

\section{The transcriptional expression of AaWRKY17 is} upregulated by Pseudomonas syringae pv. tomato DC3000 (Pst DC3000) infection and the exogenously applied phytohormones SA and MeJA

AaWRKY17 is a homologous gene of AtWRKY17 that has been proven to be involved in the response to bacterial $P$. syringae in $A$. thaliana. We inferred that AaWRKY17 might also play a role in disease resistance in A. annua. To further investigate the possible biological function of AaWRKY17 in plant disease resistance, the expression pattern of AaWRKY17 was examined after inoculation of WT A. annua with Pst DC3000. The results of RT-qPCR experiments revealed that the expression of AaWRKY17 was induced drastically upon Pst DC3000 attack compared to that in the mock-treated leaves (Fig. 5A). As shown in Fig. 5A, AaWRKY17 responded to Pst DC3000 quickly and peaked at $1 \mathrm{hpi}$ (hours post inoculation), which implied that AaWRKY17 was potentially involved in the response of $A$. annua to $P$. syringae (Fig. 5A). To further confirm that AaWRKY17 was related to the response to $P$. syringae in $A$. annua, the relative expression of AaWRKY17 under SA, MeJA, ETH, and ABA treatments was measured by RT-qPCR. The exogenous application of $1 \mathrm{mM}$ SA resulted in significantly enhanced expression of AaWRKY17 and was maintained until 12 hpt (hours post treatment) (Fig. 5B). Additionally, the transcript level of AaWRKY17 was also increased when treated with $100 \mu \mathrm{m} \mathrm{MeJA}$ at $1 \mathrm{hpt}$ (Fig. 5D). Compared to the mock treatment, however, the transcript level of AaWRKY17 showed no obvious difference with the exogenous application of ETH or ABA (Fig. 5C, E). These results indicated that the expression of $A a W R K Y 17$ was greatly induced by $P$. syringae infection and exogenously applied SA and MeJA. Hence, the TF AaWRKY17 was probably involved in the SA and MeJA signaling pathways in response to $P$. syringae in $A$. annua.

\section{AaWRKY17 functions as a positive regulator of disease resistance to $P$. syringae}

Subsequently, to further test whether AaWRKY17 functions in disease resistance, the phenotypes of 
A

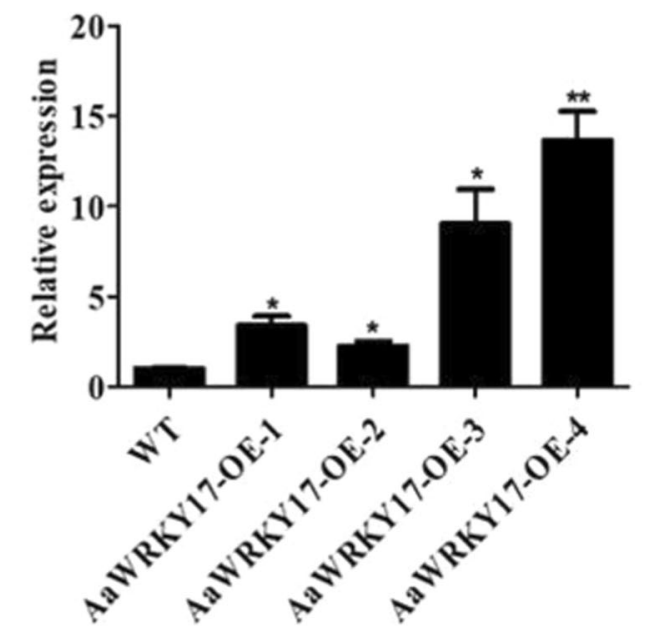

C

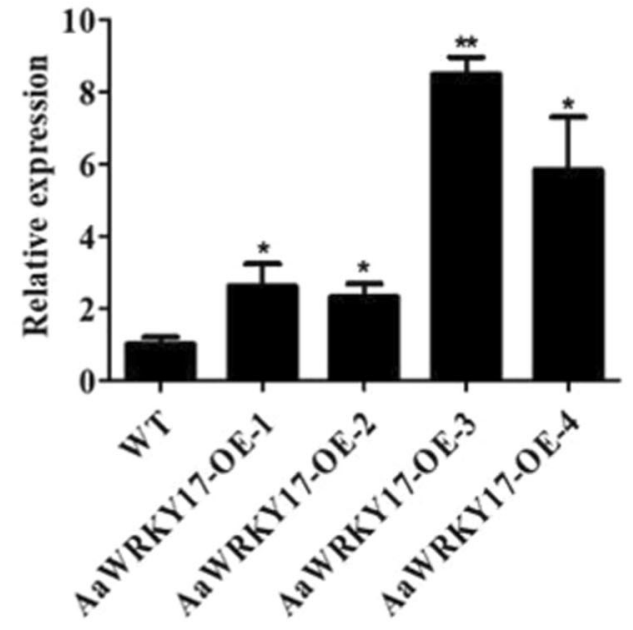

$\mathbf{E}$

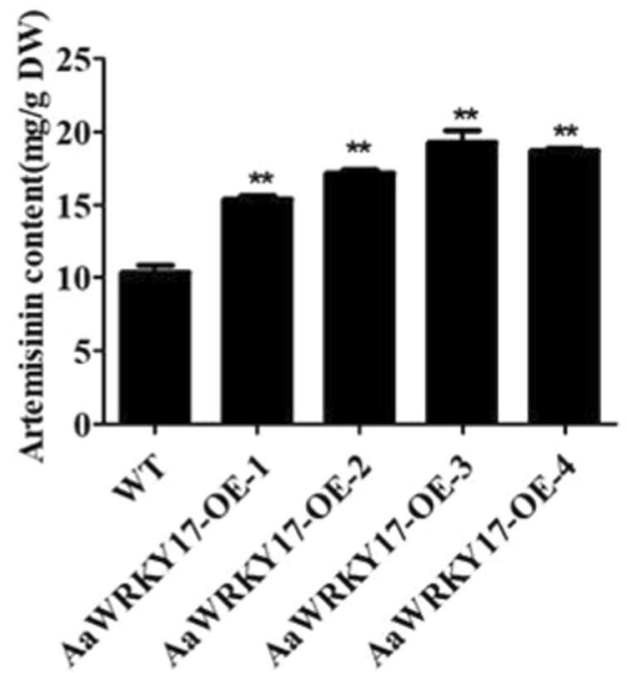

B

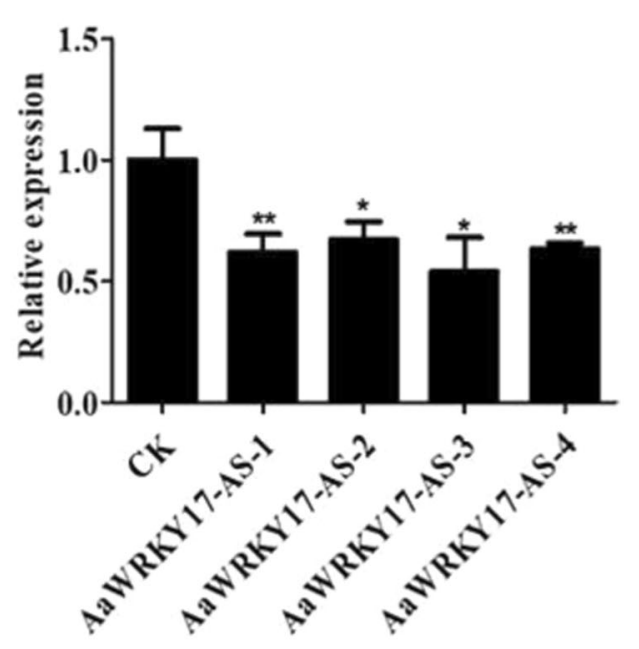

D

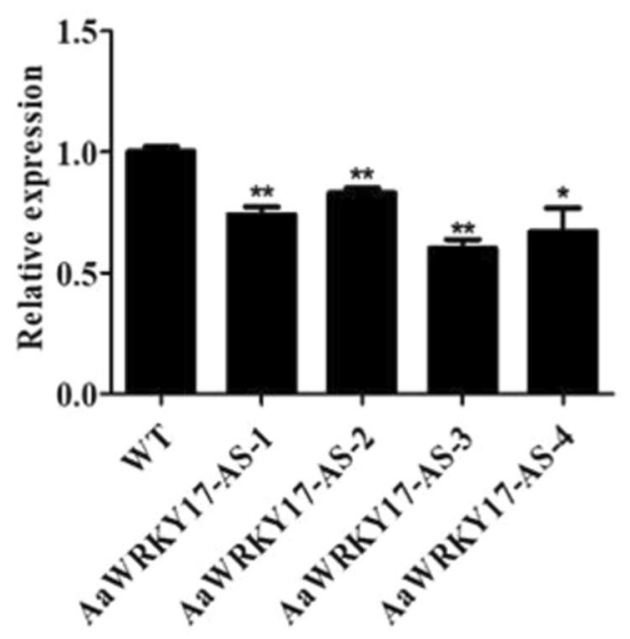

F

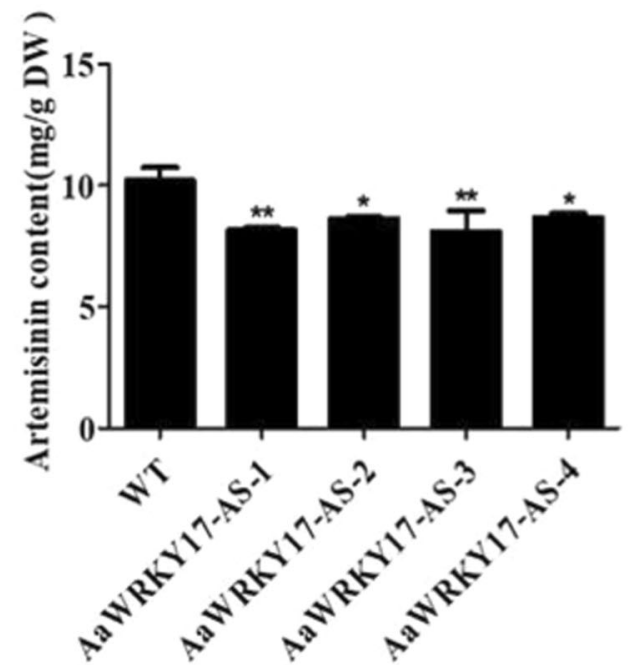

Fig. 3 Analysis of AaWRKY17 transgenic A. annua plants. Expression levels of AaWRKY17 in different AaWRKY17 overexpression (A) and antisense lines (B). Transgenic plants were measured by RT-qPCR. WT, wild-type. Actin was used as an internal reference. Expression levels of ADS in different AaWRKY17 overexpression (C) and antisense lines (D). Transgenic plants were measured by RT-qPCR. WT, wild-type. Actin was used as an internal reference. Artemisinin content in different AaWRKY17 overexpression (E) and antisense lines (F), measured by high-performance liquid chromatography (HPLC). All data are given as the means \pm SD $(n=3){ }^{*} p<0.05$; ${ }^{* *} p<0.01$; Student's $t$ test 
A

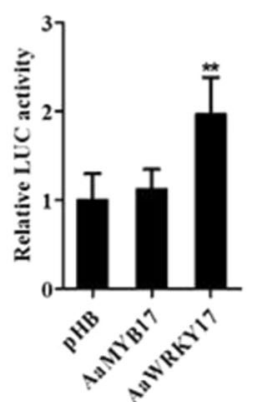

C
B

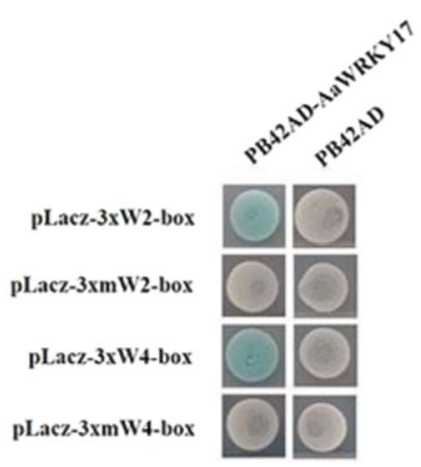

D

Shift bands

Free probe
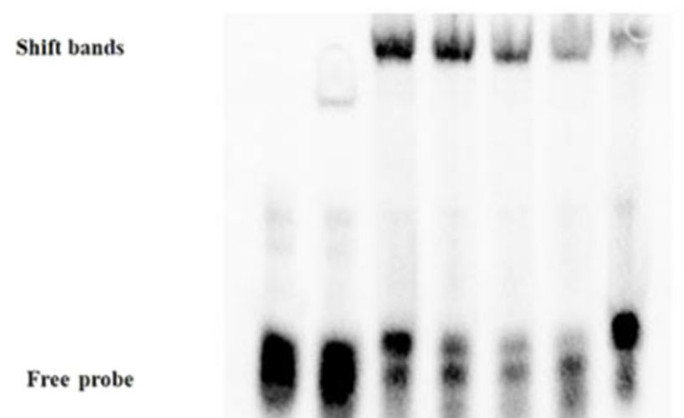

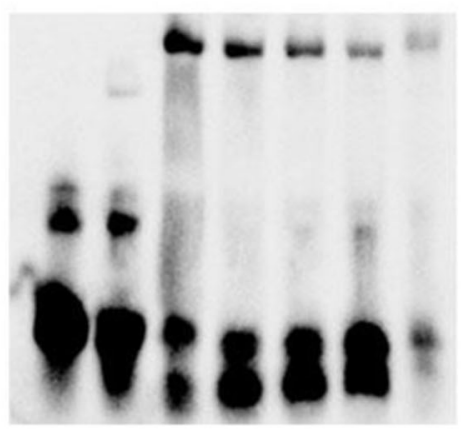

Shift bands

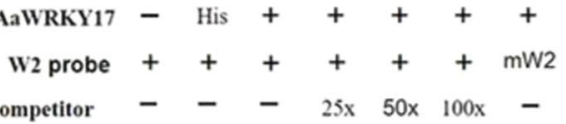

Free probe

Fig. 4 AaWRKY17 directly binds to and activates the promoter of ADS. A Effects of AaWRKY17 on ADS promoter activation. The activity of the ADS promoter fused to the LUC reporter was determined using a transient dual-LUC assay in tobacco. The relative LUC activity was normalized to the reference Renilla (REN) luciferase. Data are given as means \pm SD $(n=4)$. Student's $t$ test: ${ }^{*} p<0.05,{ }^{* *} p<0.01$. The empty vector pHB was used as a negative control. The vector pHB-AaMYB17 was used as a positive control. B Yeast one-hybrid assay of protein-DNA interactions. The empty vector pB42AD was used as a negative control. C, D Electrophoretic mobility shift assay (EMSA) showing AaWRKY17 binding to the W2 box ( -2174 to -2179 ) and W4 box ( -298 to -303 ) of the ADS promoter. Purified His-AaWRKY17 fusion protein and biotin-labeled probes were used. Nonlabeled probes $(25 \mathrm{x}, 50 \mathrm{x}, 100 \mathrm{x})$ were added as competitors. Purified His-pCold proteins were used as a negative control

AaWRKY17-overexpressing lines and WT plants following inoculation with Pst DC3000 were observed at $5 \mathrm{dpi}$ (days post inoculation). Compared to the WT plants, disease symptoms were significantly reduced in each of the AaWRKY17-overexpressing lines (Fig. 6A). All the leaves of WT plants showed different levels of infection symptoms, whereas only $13 \%$ of the leaves from AaWRKY17-overexpressing lines were symptomatic. To clearly show the difference in the infected symptoms between WT and AaWRKY17-overexpressing lines. We performed a bacterial growth assay with Pst DC3000 of infected plants. The statistical analysis results showed that less bacterial growth was observed in AaWRKY17-overexpressing plants than in WT plants (Fig. 6B). Moreover, RT-qPCR was used to check the transcriptional expression level of known defense-related marker genes NHL10 and PR5. As shown in Fig. 6C, the expression of NHL10 and PR5 was greatly increased in AaWRKY17- overexpressing lines (Fig. 6C). These results demonstrated that AaWRKY17 was a positive regulator of disease resistance to $P$. syringae in $A$. annua.

\section{Discussion}

Artemisinin and its derivatives, which are isolated from A. annua, have been proven to cure malaria efficiently. In the biosynthesis of artemisinin, ADS catalyzes the conversion of FPP (farnesyl diphosphate) to amorpha-4,11diene, which is the first step of artemisinin production. Then, amorpha-4,11-diene is converted to dihydroartemisinic acid (DHAA) with the aid of CYP71AV1, DBR2, and ALDH1. After photooxidation, DHAA is transformed to artemisinin in the glandular trichome of A. annua. Understanding the transcriptional regulation underlying artemisinin biosynthesis has remained of global concern. In recent decades, numerous TFs, such as those from the $\mathrm{TCP}^{22}, \mathrm{bHLH}^{23}, \mathrm{bZIP}^{24}$, and WRKY ${ }^{25,26}$ 
A

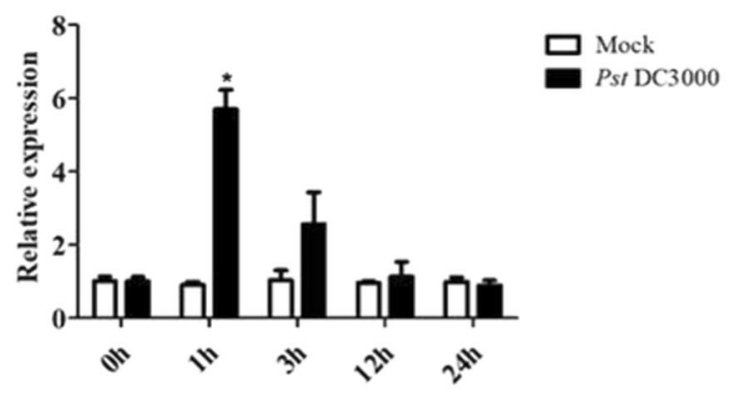

C

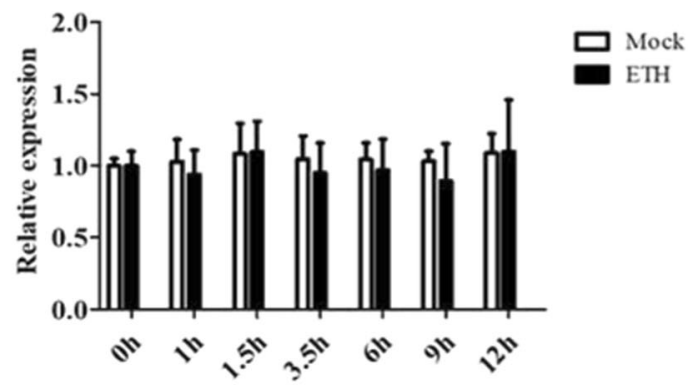

$\mathbf{E}$

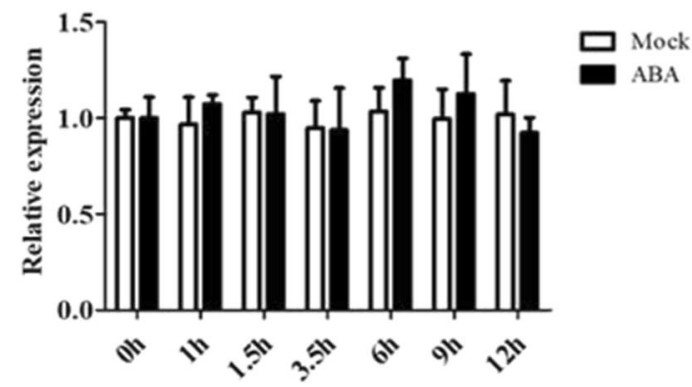

B

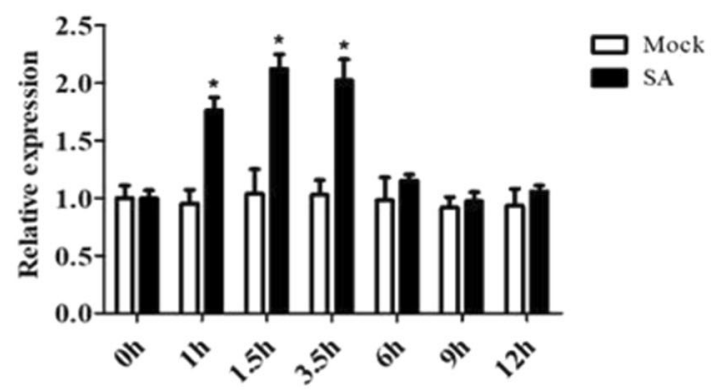

D

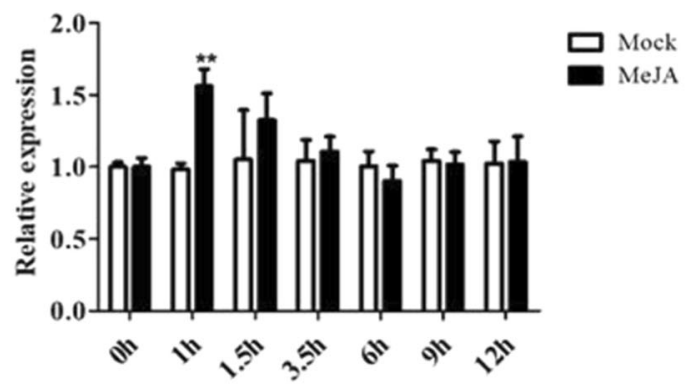

Fig. 5 Relative AaWRKY17 transcriptional levels after Pst DC3000 inoculation and different phytohormone treatments. RT-qPCR waS performed on samples with A Pst DC3000 inoculation, B application of $1 \mathrm{mM} \mathrm{SA}, \mathbf{C}$ application of $100 \mu \mathrm{m} \mathrm{MeJA,} \mathrm{D} \mathrm{application} \mathrm{of} 100 \mu \mathrm{m}$ ETH, and E application of $100 \mu \mathrm{m}$ ABA. A Plants were treated with $10 \mathrm{mM} \mathrm{MgCl}_{2}$ as a mock treatment. B, D, E Plants were treated with $0.1 \%$ ethanol as a mock treatment. C, D Plants were treated with $\mathrm{dd}_{2} \mathrm{O}$ as a mock treatment. All data are given as the means $\pm \mathrm{SD}(n=3),{ }^{*} p<0.05 ;{ }^{* *} p<0.01$; Student's $t$ test

families, have been identified to be involved in artemisinin biosynthesis. In $A$. annua, AaWRKY1 and AaGSW1 are two WRKY TFs that are reported to regulate artemisinin biosynthesis $^{25,26}$. Nevertheless, knowledge about the function of the WRKY family in artemisinin biosynthesis is far from complete. To this end, five WRKY genes that were highly expressed in the trichome of $A$. annua were selected and displayed on a heatmap based on their RPKM values (Fig. 1). Among the five candidate WRKY genes, AaWRKY17 showed the highest expression in trichomes (Figs. 2A and S1). Therefore, AaWRKY17 was chosen for generating transgenic $A$. annua plants.
Overexpression of $A a W R K Y 17$ resulted in enhanced artemisinin content, whereas antisense-based gene silencing of AaWRKY17 decreased artemisinin content in $A$. annua (Fig. 3E, F). In addition, the transcript level of the artemisinin biosynthesis pathway structural gene $A D S$ was significantly increased in AaWRKY17-overexpressing lines (Fig. 3C, D). To further study the transcriptional regulation of AaWRKY17, dual-LUC, Y1H, and EMSA were carried out. As shown in Fig. 5, AaWRKY17 can directly bind to and activate the promoter of $A D S$ in vivo and in vitro. Hence, we confirmed that AaWRKY17 was a positive regulator of artemisinin biosynthesis in $A$. annua. 


\section{$\mathbf{A}$}

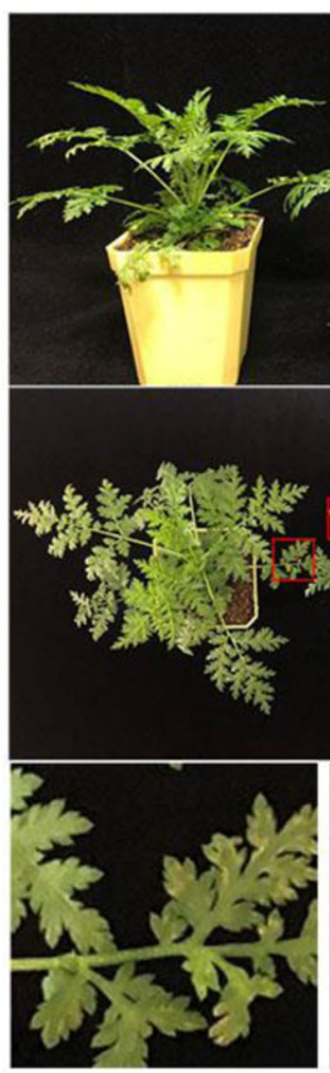

WT

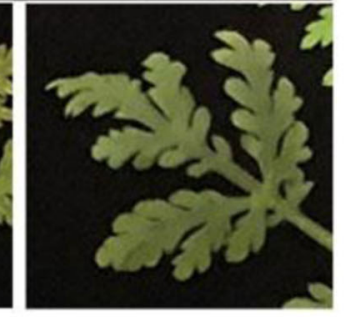

AaWRKY17-OE-4
B

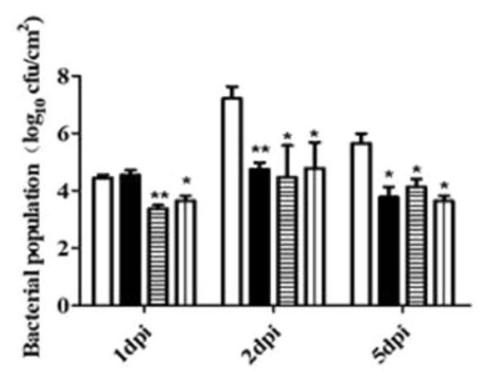

C

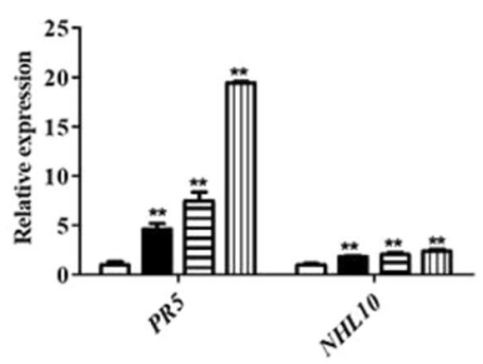

ㅁ WT

AaWRKY17-OE-1 AaWRKY17-OE-2 血 AaWRKY17-OE-4

Fig. 6 AaWRKY17 functions as a positive regulator of disease resistance to Pseudomonas syringae. A Phenotypes of WT and AaWRKY17overexpressing transgenic lines at $5 \mathrm{dpi}$. B Bacterial population at 1,2, and $5 \mathrm{dpi}$ in WT- and AaWRKY17-overexpressing transgenic lines. Data are given as the means $\pm \mathrm{SD}(n=3),{ }^{*} p<0.05 ;{ }^{* *} p<0.01$; Student's $t$ test. cfu colony forming units. C RT-qPCR analysis of transcriptional levels of defenserelated marker genes in WT and AaWRKY17-overexpressing transgenic lines. Data are given as the means $\pm \operatorname{SD}(n=3),{ }^{*} p<0.05 ;{ }^{* *} p<0.01$; Student's $t$ test

In addition to artemisinin biosynthesis, the role of AaWRKY17 in regulating the biotic stress of A. annua was also investigated. Plants are subjected to a variety of biotic stresses during their whole life. Thus, plants have developed a multitude of defense mechanisms to face these stresses, including mechanisms against pathogen stress. As artemisinin is the most promising drug for the treatment of malaria, cancer, and tuberculosis, producing high-yielding $A$. аnnua is important for meeting the high demand for artemisinin. In contrast to the study of the artemisinin biosynthetic pathway, there is much less information about the effects of different pathogens on $A$. annua. A previous study showed that the trichome-specific AP2/ERF transcription factor AaORA can positively regulate artemisinin biosynthesis and resistance to B. cinerea in $A$. annua ${ }^{34}$. Moreover, another AP2/ERF transcription factor, AaERF1, was reported to regulate resistance to $B$. cinerea in $A$. annua $^{35}$. In addition to AP2/ERF TFs, AaNAC1 was found to enhance artemisinin content as well as tolerance to drought and B. cinerea in transgenic A. annua plants $^{36}$. However, far too little attention has been given to $P$. syringae, which causes economically important diseases in a wide range of plant species. It has previously been observed that the trichomes of plants have a close relationship with disease resistance ${ }^{37}$ and that AtWRKY17 is involved in the response to P. syringae in A. thaliana ${ }^{38}$. As a homologous gene of AtWRKY17, we inferred that $A a W R K Y 17$ may have a similar function in response to $P$. syringae in $A$. annua. RT-qPCR results showed that the transcript levels of the defense marker genes PR5 and NHL10 were increased significantly in AaWRKY17-overexpressing lines. After inoculation with Pst DC3000, compared to the WT plants, most of the AaWRKY17-overexpressing lines grew well (Fig. 6). These results demonstrated that AaWRKY17 is a positive regulator of disease resistance to $P$. syringae in $A$. aпnua. 
Additionally, the expression of AaWRKY17 was induced drastically by the exogenous application of SA and MeJA (Fig. 5). The plant hormones JA and SA have been reported as positive regulators of artemisinin biosynthesis in $A$. апии $a^{39,40}$. Furthermore, many pathogen-responsive genes are regulated in an SA- or JA-dependent manner. For instance, WRKY62 was recently reported to have a putative role in modulating the cross-talk between the SA and JA signaling pathways ${ }^{41}$. A. thaliana mutant coil showed enhanced susceptibility to Pst DC3000 due to the interference of SA synthesis or SA signal transduction, whereas the mutants that were defective in JA signal transduction possessed elevated resistance to Pst DC3000 ${ }^{42}$. Taken together, our study revealed a new WRKY TF, AaWRKY17, that has dual functions in artemisinin biosynthesis and disease resistance in A. annua.

\section{Materials and methods}

\section{Plant materials}

The $A$. annua cultivar used in this study was "Huhao 1", which had a high artemisinin content of $8-10 \mathrm{mg} / \mathrm{g}$ and was originally obtained from the School of Life Sciences, Southwest University, Chongqing ${ }^{23}$. A. annua and tobacco (Nicotiana benthamiana) were grown under a $16 \mathrm{~h}$ photoperiod at $24 \pm 2{ }^{\circ} \mathrm{C}$.

\section{Subcellular localization}

For subcellular localization, the ORF of AaWRKY17 was amplified by PCR and then cloned into the plant expression vector pHB-YFP. The construct was transferred into A. tumefaciens strain GV3101 and transiently transformed into 4-week-old tobacco leaves. YFP signals were observed by confocal laser microscopy after $48 \mathrm{~h}$ of weak light exposure. Nuclei were stained with 4',6-diamidino-2-phenylindole staining, and pHB-YFP was used as a negative control. All primers are listed in Supplementary Table S1.

\section{Hormone treatments}

For hormone treatments, 15-day-old WT A. апnиa were sprayed with $100 \mu \mathrm{M}$ MeJA, $100 \mu \mathrm{M}$ ETH, $100 \mu \mathrm{M}$ $\mathrm{ABA}$, and $1 \mathrm{mM} \mathrm{SA}$. For the mock MeJA, ABA, and SA treatment, seedlings were sprayed with $0.1 \%$ ethanol. For the mock ETH treatment, the seedlings were sprayed with $\mathrm{ddH}_{2} \mathrm{O}$. The growth point (GP) and leaf 0 of the seedlings were collected at $0,1,1.5,3.5,6,9$, and $12 \mathrm{hpt}$ for RTqPCR analysis. Three biological repeats were performed to verify these results.

\section{RNA extraction and real-time quantitative polymerase chain reaction (RT-qPCR)}

To detect the transcript level of candidate genes in different tissues and leaves at different positions in $A$. апnиа, tissue samples (stem, old leaves, bud 0, flower, root, shoot, young leaves, and trichome) and leaves at different positions (GP, leaf 0 , leaf 1 , leaf 2 , leaf 3 , leaf 4 , leaf 5 , and leaf 6) were collected as described previously $^{17}$. To analyze the expression of AaWRKY17, $A D S$, PR5, and NHL10 in AaWRKY17 transgenic A. annua plants, samples of GP and leaf 0 of a single plant were selected. The total RNA of all samples was extracted using a plant RNA isolation reagent (Tiangen Biotech, Beijing, China), and cDNA was synthesized by using PrimeScript ${ }^{\mathrm{TM}}$ RT Master Mix (Takara, Shiga, Japan). Expression levels were normalized to the ACTIN control gene (GenBank accession number EU531837) ${ }^{39}$ and calculated by the $2^{-\boldsymbol{\Lambda} \mathbf{\Delta} \mathrm{CT}}$ method (ref. ${ }^{43}$ ). Three independent biological replicates were performed, each containing three technical replicates. All primers are listed in Supplementary Table S1.

\section{A. annua transformation}

The constructs pHB-AaWRKY17-YFP, pHB-AaWRKY17antisense, and 1391Z-proAaWRKY17-GUS were introduced into the Agrobacterium tumefaciens strain EHA105 and then used to transform A. annua as described previously ${ }^{44}$.

\section{Artemisinin content measurement}

Five-month-old $A$. annua leaves were gathered to measure the artemisinin content as described previously ${ }^{17}$. All the $A$. annua leaves were ground to a powder after drying in a $50{ }^{\circ} \mathrm{C}$ air oven for $24 \mathrm{~h}$. An aliquot of $0.1 \mathrm{~g}$ dry powder was extracted using $1 \mathrm{ml}$ methanol under sonication for $30 \mathrm{~min}$ at $55 \mathrm{~Hz}$ and then centrifuged for $10 \mathrm{~min}$ at $12,000 \mathrm{rpm}$. The $1 \mathrm{ml}$ supernatant was collected in a new tube. The above steps were repeated, and $2 \mathrm{ml}$ supernatant was collected for the next analysis. The artemisinin content was measured by HPLC $^{45}$. Three biological repeats were measured.

\section{Dual-luciferase (dual-LUC) assay in tobacco leaves}

For the dual-LUC assays, the ORF of AaWRKY17 was cloned into the pHB vector as an effector, and the promoter of $A D S$ was cloned into the pGreenII 0800-LUC vector as a reporter ${ }^{46}$. The effector and reporter were transformed into A. tumefaciens strain GV3101 and A. tumefaciens strain GV3101, respectively, with the helper plasmid pSoup 19. The pHB empty vector was used as a negative control, and the Renilla luciferase (REN) gene under the control of the constitutive $35 \mathrm{~S}$ promoter was used as an internal reference. AaMYB17, which was reported not to regulate the expression of $A D S$, was used as a control ${ }^{47}$. Different combinations of the effectors and reporter were mixed in a 9:1 volume ratio to transform 4week-old tobacco leaves. Leaves were collected after $48 \mathrm{~h}$ of incubation under weak light to measure the LUC and REN activities using commercial dual-LUC reaction reagents (Promega). Four biological repeats were 
performed for each combination. All primers are listed in Supplementary Table S1.

\section{Yeast one-hybrid (Y1H) assay}

Yeast one-hybrid assays were conducted as previously described $^{26}$. The ORFs of AaWRKY17 were amplified and ligated into the pB42AD vector, and the AaWRKY17 binding sites (W-box) of the $A D S$ promoter were cloned into the pLacZ vector. These plasmids were cotransformed into yeast strain EGY48A, which was cultivated on $\mathrm{SD} /$-Ura/-Leu medium for $72 \mathrm{~h}$ and tested on $\mathrm{SD} /$-Ura/Leu medium with 5-bromo-4-chloro-3-indolyl-b-Dgalactopyranoside (X-gal) for $48 \mathrm{~h}$. The empty pB42AD and pLacZ plasmids were used as negative controls. All primers are listed in Supplementary Tables S1 and S2.

\section{Electrophoretic mobility shift assay (EMSA)}

For protein expression and purification, the pColdAaWRKY17 vector was constructed and transformed into Escherichia coli strain Rosetta (DE3) (TransGen Biotech, China). Heterologous protein production was induced by adding $0.5 \mathrm{mM}$ isopropyl $\beta$-D-1-thiogalactopyranoside to the bacterial culture for $16 \mathrm{~h}$ at $16^{\circ} \mathrm{C}$ and purified using HisSep Ni-NTA (nitrilotriacetic) agarose resin (Yeasen, China). The W2-box, W4-box, mW2-box, and mW4-box probes from the promoter of $A D S$ were synthesized and then labeled with biotin at their $5^{\prime}$ end by Sangon (Shanghai, China). EMSAs were performed using the LightShift Chemiluminescent EMSA Kit (Thermo, USA) following the manufacturer's instructions. The probes used in the EMSA are listed in Supplementary Table S2.

\section{Pseudomonas syringae pv. tomato DC3000 (Pst DC3000) inoculation and quantification}

Six-week-old $A$. аnnua plants were used for bacterial inoculation. Briefly, Pst DC3000 was cultivated at $28^{\circ} \mathrm{C}$ and $200 \mathrm{rpm}$ in King's medium B with rifampicin $(50 \mathrm{mg} /$ l), collected by centrifugation, resuspended in $10 \mathrm{mM}$ $\mathrm{MgCl}_{2}$ at $\mathrm{OD}_{600}=0.6$ and supplemented with $0.04 \%$ Silwet L-77 for spray inoculation ${ }^{48}$. After Pst DC3000 inoculation, the plants were kept at $100 \%$ relative humidity. The GP and leaf 0 were sampled at $0,1,3,6,12$, and 24 hpi for RT-qPCR. For quantification of bacterial populations, six leaves $\left(10 \mathrm{~mm}^{2} \times 6\right)$ from one individual plant were collected at 1,2 , and $5 \mathrm{dpi}$, washed twice with sterile water and homogenized in King's B containing rifampicin, followed by $10^{-1}, 10^{-2}, 10^{-3}, 10^{-4}, 10^{-5}$, and $10^{-6}$ dilutions on solid medium.

\section{Acknowledgements}

This work was supported by the National Key R\&D Program of China (2018YFA0900600), the Bill \& Melinda Gates Foundation (OPP1199872 and INV027291), SJTU Trans-med Awards Research (20190104) and the SJTU Global Strategic Partnership Fund (2020 SJTU-CORNELL).

\section{Author contributions}

T.C., L.X., and K.T. designed the project; T.C., Y.L., W.Q., X.F., C.W., X.Y., and Y.Z. performed most of the experiments; B.P., L.L., XH.Y., L.X., X.H., K.W-Z., and K.T. analyzed the data and discussed the article; T.C. and Y.L. wrote the manuscript. All the authors have read and approved the manuscript.

Conflict of interest

The authors declare no competing interests.

Supplementary information The online version contains supplementary material available at https://doi.org/10.1038/s41438-021-00652-6.

Received: 13 April 2021 Revised: 8 June 2021 Accepted: 13 June 2021 Published online: 01 October 2021

\section{References}

1. Suzuki, N., Rivero, R. M., Shulaev, V., Blumwald, E. \& Mittler, R. Abiotic and biotic stress combinations. N. Phytol. 203, 32-43 (2014).

2. Chisholm, S. T., Coaker, G., Day, B. \& Staskawicz, B. J. Host-microbe interactions: shaping the evolution of the plant immune response. Cell 124, 803-814 (2006).

3. Atkinson, N. J. \& Urwin, P. E. The interaction of plant biotic and abiotic stresses: from genes to the field. J. Exp. Bot. 63, 3523-3543 (2012).

4. Bakshi, M. \& Oelmüller, R. WRKY transcription factors jack of many trades in plants. Plant Signal Behav. 9, 1-18 (2014).

5. Pandey, S. P. \& Somssich, I. E. The role of WRKY transcription factors in plant immunity. Plant Physiol. 150, 1648-1655 (2009).

6. Katagiri, F. \& Tsuda, K. Understanding the plant immune system. Mol. PlantMicrobe Interact. 23, 1531-1536 (2010).

7. Eulgem, T. \& Somssich, I. E. Networks of WRKY transcription factors in defense signaling. Curr. Opin. Plant Biol. 10, 366-371 (2007).

8. Rushton, P. J., Somssich, I. E., Ringler, P. \& Shen, Q. J. WRKY transcription factors. Trends Plant Sci. 15, 247-258 (2010).

9. Jiang, J. et al. WRKY transcription factors in plant responses to stresses. J. Integr. Plant Biol. 59, 86-101 (2017).

10. Xu, X., Chen, C., Fan, B. \& Chen, Z. Physical and functional interactions between pathogen-induced Arabidopsis WRKY18, WRKY40, and WRKY60 transcription factors. Plant Cell. 18, 1310-1326 (2006).

11. Birkenbihl, R. P., Diezel, C. \& Somssich, I. E. Arabidopsis WRKY33 is a key transcriptional regulator of hormonal and metabolic responses toward Botrytis cinerea infection. Plant Physiol. 159, 266-285 (2012).

12. Chen, $\mathrm{X}$. et al. Overexpression of AtWRKY28 and AtWRKY75 in Arabidopsis enhances resistance to oxalic acid and Sclerotinia sclerotiorum. Plant Cell Rep. 32, 1589-1599 (2013).

13. Hussain, A. et al. CaWRKY22 acts as a positive regulator in pepper response to Ralstonia solanacearum by constituting networks with CaWRKY6, CaWRKY27, CaWRKY40, and CaWRKY58. Int J. Mol. Sci. 5, 1426-1444 (2018).

14. Chen, L., Zhang, L. \& Yu, D. Wounding-Induced WRKY8 is involved in basal defense in Arabidopsis. Mol. Plant-Microbe Interact. 23, 558-565 (2010).

15. Lai, Z., Vinod, K., Zheng, Z., Fan, B. \& Chen, Z. Roles of Arabidopsis WRKY3 and WRKY4 transcription factors in plant responses to pathogens. BMC Plant Biol. 8, $1-14$ (2008).

16. Yan, T. et al. A novel HD-ZIP IV/MIXTA complex promotes glandular trichome initiation and cuticle development in Artemisia annua. N. Phytol. 218, 567-578 (2018).

17. Xie, L. et al. The WRKY transcription factor AaGSW2 promotes glandular trichome initiation in Artemisia annua. J. Exp. Bot. 72, 1691-1701 (2020).

18. White, N. J. \& Properties, P. Review qinghaosu (Artemisinin): the price of success. Science 320, 330-335 (2008).

19. Leann, T. et al. Artemisinin action and resistance in Plasmodium falciparum Trends Parasitol. 32, 682-696 (2016).

20. Efferth, T. From ancient herb to modern drug: Artemisia annua and artemisinin for cancer therapy. Semin. Cancer Biol. 46, 65-83 (2017).

21. Brown, G. D. \& Sy, L. K. In vivo transformations of dihydroartemisinic acid in Artemisia annua plants. Tetrahedron 60, 1139-1159 (2004).

22. $\mathrm{Ma}$, Y. et al. Jasmonate promotes artemisinin biosynthesis by activating the TCP14-ORA complex in Artemisia annua. Sci. Adv. 4, 1-19 (2018). 
23. Shen, Q. et al. The jasmonate-responsive AaMYC2 transcription factor positively regulates artemisinin biosynthesis in Artemisia annua. N. Phytol. 210, 1269-1281 (2016).

24. Hao, X. et al. Light-induced artemisinin biosynthesis is regulated by the bZIP transcription factor AaHY5 in Artemisia annua. Plant Cell Physiol. 60, 1747-1760 (2019).

25. Ma, D. et al. Isolation and characterization of AaWRKY1, an Artemisia annua transcription factor that regulates the amorpha-4,11-diene synthase gene, a key gene of artemisinin biosynthesis. Plant Cell Physiol. 50, 2146-2161 (2009).

26. Chen, M. et al. Glandular trichome-specific WRKY 1 promotes artemisinin biosynthesis in Artemisia annua. N. Phytol. 214, 304-316 (2017).

27. Niu, D. D. et al. The plant growth-promoting rhizobacterium bacillus cereus AR156 induces systemic resistance in Arabidopsis thaliana by simultaneously activating salicylate- and jasmonate/ethylene-dependent signaling pathways. Mol. Plant-Microbe Interact. 24, 533-542 (2011).

28. Pieterse, C., Leon-Reyes, A., Van, S. \& Van, W. Networking by small-molecule hormones in plant immunity. Nat. Chem. Biol. 5, 308-316 (2009).

29. Birkenbihl, R. P., Liu, S. \& Somssich, I. E. Transcriptional events defining plant immune responses. Curr. Opin. Plant Biol. 38, 1-9 (2017).

30. Banerjee, A. \& Roychoudhury, A. WRKY proteins: signaling and regulation of expression during abiotic stress responses. Sci. World J. 2015, 807560 (2015).

31. Cen, C. \& Chen, Z. Potentiation of developmentally regulated plant defense response by AtWRKY18, a pathogen-induced Arabidopsis transcription factor. Plant Physiol. 129, 706-716 (2002).

32. Liu, F. et al. N-3-oxo-octanoyl-homoserine lactone-mediated priming of resistance to Pseudomonas syringae requires the salicylic acid signaling pathway in Arabidopsis thaliana. BMC Plant Biol. 20, 38 (2020).

33. Po-Wen, C., Singh, P. \& Zimmerli, L. Priming of the Arabidopsis patterntriggered immunity response upon infection by necrotrophic Pectobacterium carotovorum bacteria. Mol. Plant Pathol. 14, 58-70 (2013).

34. Lu, X. et al. AaORA, a trichome-specific AP2/ERF transcription factor of Artemisia annua, is a positive regulator in the artemisinin biosynthetic pathway and in disease resistance to Botrytis cinerea. N. Phytol. 198, 1191-1202 (2013).

35. Lu, X. et al. AaERF1 positively regulates the resistance to Botrytis cinerea in Artemisia annua. PLoS ONE 8, e57657 (2013)

36. LV, Z. et al. Overexpression of a novel NAC domain-containing transcription factor gene $(A a N A C 1)$ enhances the content of artemisinin and increases tolerance to drought and botrytis cinerea in Artemisia annua. Plant Cell Physiol. 57, 1961-1971 (2016)

37. Schilmiller, A. L., Last, R. L. \& Pichersky, E. Harnessing plant trichome biochemistry for the production of useful compounds. Plant J. 54, 702-711 (2008).

38. Journot-Catalino, H., Somssich, I. E., Roby, D. \& Kroj, T. The transcription factors WRKY11 and WRKY17 act as negative regulators of basal resistance in Arabidopsis thaliana. Plant Cell. 18, 3289-3302 (2006).

39. Maes, L. et al. Dissection of the phytohormonal regulation of trichome formation and biosynthesis of the antimalarial compound artemisinin in Artemisia annua plants. N. Phytol. 189, 176-189 (2011).

40. LV, Z., Zhang, L. \& Tang, K. New insights into artemisinin regulation. Plant Signal Behav. 12, e1366398 (2017).

41. Mao, P., Duan, M., Wei, C. \& Li, Y. WRKY62 transcription factor acts downstream of cytosolic NPR1 and negatively regulates jasmonate-responsive gene expression. Plant Cell Physiol. 48, 833-842 (2007).

42. Kloek, A. P. et al. Resistance to Pseudomonas syringae conferred by an Arabidopsis thaliana coronatine-insensitive (coi1) mutation occurs through two distinct mechanisms. Plant J. 26, 509-522 (2001).

43. Livak, K. J. \& Schmittgen, T. D. Analysis of relative gene expression data using real-time quantitative $P C R$ and the 2- $\triangle \triangle C T$ method. Methods 25, 402-408 (2001).

44. Zhang, L. et al. Development of transgenic Artemisia annua (Chinese wormwood) plants with an enhanced content of artemisinin, an effective antimalarial drug, by hairpin-RNA-mediated gene silencing. Biotechnol. Appl. Biochem. 52, 199 (2009).

45. Li, Y. et al. Transcriptomic analysis reveals the parallel transcriptional regulation of UV-B-induced artemisinin and flavonoid accumulation in Artemisia annua L. Plant Physiol. Biochem. 163, 189-200 (2021).

46. Hellens, R. P. et al. Transient expression vectors for functional genomics, quantification of promoter activity and RNA silencing in plants. Plant Methods 1, 1-14 (2005).

47. Qin, W. et al. An R2R3-MYB transcription factor positively regulates the glandular secretory trichome initiation in Artemisia annua L. Front. Plant Sci. 12, 1-10 (2021).

48. Katagiri, F., Thilmony, R. \& He, S. Y. The Arabidopsis Thaliana-Pseudomonas Syringae interaction. Arab B. 1, e0039 (2002) 